Voix et Images

voixetimages

\title{
Janet M. Paterson, Anne Hébert : Architexture romanesque
}

\section{Alain Piette}

Volume 12, numéro 1 (34), automne 1986

Québec-Amérique latine

URI : https://id.erudit.org/iderudit/200613ar

DOI : https://doi.org/10.7202/200613ar

Aller au sommaire du numéro

Éditeur(s)

Université du Québec à Montréal

ISSN

0318-9201 (imprimé)

1705-933X (numérique)

Découvrir la revue

Citer cet article

Piette, A. (1986). Janet M. Paterson, Anne Hébert : Architexture romanesque.

Voix et Images, 12(1), 124-126. https://doi.org/10.7202/200613ar d'utilisation que vous pouvez consulter en ligne.

https://apropos.erudit.org/fr/usagers/politique-dutilisation/ 


\title{
Janet M. Paterson, Anne Hébert: Architexture romanesque ${ }^{1}$
}

\author{
par Alain Piette, Université du Québec à Montréal
}

Cet essai se classe parmi les travaux de type universitaire: il correspond aux lois du genre de par ses notes infrapaginales, l'exhaustivité de sa démonstration, ses tableaux et relevés, etc. Loin d'être péjorative, cette précision veut simplement situer les enjeux de départ d'une recherche qui, en plus d'être rigoureuse, stimule le lecteur et lui donne le goût de retourner au corpus hébertien. Il existe une dizaine d'ouvrages sur Anne Hébert, mais celui-ci n'a rien à envier à ses prédécesseurs: il occupe un espace laissé à peu près vacant jusque-là. Tout d'abord, c'est la première fois qu'un long texte est consacré aux Chambres de bois (la plus grande partie du présent essai). De plus, le type d'analyse textuelle pratiqué ici s'écarte d'une bonne partie des productions antérieures où trop souvent la seule thématique était ressassée. On pourrait dire que cela rajeunit la critique hébertienne en quelque sorte. Enfin, l'auteure a su démontrer la pertinence d'étiquettes apposées depuis longtemps sur l'œuvre d'Anne Hébert par une critique sagace mais trop impressionniste: ainsi la dimension onirique et la polysémie de cette œuvre en particulier seront-elles ici mises en évidence.

Architexture romanesque: titre inspiré s'il en est, pour un essai qui entend démonter les rouages de la machine textuelle. Titre à valeur iconique aussi où la mutation d'une lettre souligne habilement l'aspect signifiant dont s'occupe constamment l'essayiste. De façon très astucieuse la recherche est amorcée à partir d'une analyse de l'incipit du roman et le mot «pays" sert de déclencheur: c'est en effet l'étude des occurrences de ce lexème dans le roman qui permettra d'établir l'existence de trois codes qui structurent le niveau sémantique du texte: codes du réel, de l'onirique et de l'irréel. Les manifestations de chaque code seront passées en revue, mais sans que jamais leur interrelation ne soit oubliée: la dynamique qui joue dans cette organisation textuelle apparaît ainsi comme une des composantes essentielles de ce corpus et justifie pleinement le choix du titre de cette partie: "La production du sens dans le texte». "Le texte comme inscription du sens" formera le deuxième volet de ce diptyque: après la représentation du texte, son autoreprésentation. Seront envisagés tous les procédés illustrant la réflexivité du texte: choix lexicaux, mises en abyme, figurations, métaphores textuelles, intertextualité. On trouve ici des analyses fines (les meilleures de l'ouvrage avec le chapitre sur les Fous de Bassan) qui mettent en valeur la structure plurielle du corpus hébertien. En particulier, le chapitre sur les métaphores textuelles est une réussite digne de mention. La dernière partie de l'ouvrage porte sur les autres romans. La même grille d'analyse leur est appliquée de telle sorte qu'on peut vérifier du même coup la pertinence de cette grille et la cohérence de ce corpus. Les Fous de Bassan jouissent d'un traitement particulier à la fois par la longueur de l'étude et par les réaménagements apportés à la grille d'analyse. 
Il ne faudrait pas croire toutefois que j'adhère sans réserve au beau texte de Paterson. Je tiens à formuler une critique sérieuse sur la typologie de la première partie, soit la division tripartite mentionnée plus haut: le réel, l'onirique et l'irréel. J'avoue ne pas avoir été convaincu de la nécessité d'une distinction entre l'onirique et l'irréel. En premier lieu, au niveau du sens commun, réel et irréel s'opposent et le rêve, de ce point de vue, est senti comme un phénomène hors du réel, pas moins irréel qu'un conte de fée. Dans les catégories littéraires - et c'est là que se situe l'auteure principalement - on peut opposer l'effet de réel (des romans réalistes) à l'effet d'irréel (des contes de fée). À ce propos, le rêve littéraire occupe une position mitoyenne: pour autant qu'il veut simuler le rêve réel (tel que le lecteur a pu l'expérimenter), il peut être l'objet d'effets de réel, ce qu'a bien vu l'auteure dans le corpus hébertien:

La répétition lyrique de ce récit souligne non seulement la composante textuelle, mais produit pour le lecteur une mimésis onirique, puisque le texte simulant l'activité du rêve raconte et répète, au moyen de bribes et d'échos, une histoire un peu étrange, un peu vague, mais toujours la même.

En revanche, le rêve littéraire, par des marques souvent évidentes, ne cesse de désigner son éloignement du réel, son caractère encore plus fictif que la fiction elle-même. Aussi, au plan littéraire même, retrouve-t-on la même dichotomie (réel/irréel) que suggérait le sens commun et l'on reste perplexe devant l'énoncé suivant de l'auteure:

le texte engage notre lecture dans un mouvement circulaire où récit, fiction et rêve se fondent et se confondent, où, récusant toute délimitation spatiale, temporelle et sémantique, le discours de l'irréel subvertit les contraintes d'une écriture du réel.

Le début de la phrase réitère la division tripartite sous une forme différente, mais la fin établit implicitement la division bipartite. Qu'est-ce à dire?

Peut-être la psychanalyse aurait-elle pu fournir une réponse encore plus convaincante à ce problème? Depuis longtemps en effet (depuis Freud) a-t-on reconnu la similarité du travail de l'inconscient dans les rêves nocturnes et dans les rêves diurnes, fussent-ils littéraires, l'élaboration secondaire ne faisant ici que complexifier l'objet des processus primaires. Mais c'est justement sur ce terrain que n'a pas voulu s'aventurer l'auteure. Quelques allusions timides, comme celle de la page 28 , révélant une certaine connaissance de la psychanalyse, parfois des étiquettes provenant du vocabulaire psychanalytique, mais apposées de façon plus ou moins pertinente comme c'est le cas de contenu manifeste/contenu latentà la page 57, enfin une bibliographie pour le moins bizarre dans un texte qui parle autant de l'onirique (un texte de Le Galliot et de Leclaire, deux de Bellemin-Noël, aucun de Freud qui n'est évidemment que l'inventeur de la théorie et de la méthode psychanalytiques!). Je ne parle de cette lacune que dans la mesure où la psychanalyse aurait pu corriger la typologie de la première partie de l'ouvrage. 
Dans l'ensemble, c'est plutôt la solidité au plan théorique que l'on peut observer chez Paterson. Corpus théorique consistant où l'on retrouve, cités à maintes reprises, les noms de Barthes, Todorov, Lotman et, de façon ponctuelle, ceux de Jakobson, Dällenbach, Derrida et Ricardou. Remarquable, la synthèse opérée par l'auteure à partir d'ouvrages qui auraient pu donner un mélange hétéroclite, mais elle a su justement éviter cet écueil en assurant des dominantes (je pense en particulier à Lotman et à Barthes sur un fond hjelmslevien). Ces sources théoriques sont le plus souvent utilisées avec discernement et réaménagées au besoin : par exemple la méthode pratiquée dans le chapitre «La poétique d'un mot» évoque celle de Barthes dans $\mathbf{S} / \mathbf{Z}$ (le "pas à pas" et les codes) mais l'appareil a été ici simplifié avec bonheur. Distanciée aussi la référence à Ricardou (p. 102-104): dans ce cas-ci, c'est la théorie de l'autoreprésentation même qui est plus nuancée et qui échappe donc à une dichotomie un peu naïve ainsi qu'à une conception mécanique des procédés. Paterson démontre bien en effet que l'ensemble des procédés d'autoreprésentation forme un réseau serré dans les Chambres de bois, réseau qui n'exclut pas certains procédés de représentation ou effets de réel.

Il arrive toutefois que la distance prise vis-à-vis des sources ne soit pas suffisante. La définition de la connotation fournie à la page 26 (sursignification tributaire d'une corrélation immanente au texte) reflète bien fidèlement celle de Barthes dans $\mathbf{S} / \mathbf{Z}$, peut-être trop. Il aurait fallu retourner à la définition même de Hjelmslev qui semble mettre davantage l'accent sur l'aspect social de la connotation (les niveaux de langue par exemple), sur l'aspect codé par conséquent, antérieurement à toute corrélation immanente au texte. La notion de "sème» aussi fait problème: Barthes retient la définition des sémanticiens (unité minimale de signification), mais il l'applique de façon plutôt approximative dans $\mathbf{S} / \mathbf{Z}$ comme l'auteure du présent ouvrage. Du point de vue de la sémantique, les expressions suivantes ne sont sans doute pas employées pertinemment: les sémèmes du conte (p. 31), les sémèmes d'obscurité, de clôture et de pourriture (p. 85), oppositions sémantiques (...) travail/immobilité (p. 88).

On aura compris, je l'espère, que les critiques ponctuelles exprimées ici n'affectent pas le jugement porté sur l'essentiel de l'entreprise et de la réalisation de Janet $M$. Paterson. Il y a là en effet une mise au jour de procédés divers du texte hébertien, mise au jour qui requérait, en plus des qualités habituelles du chercheur, une écoute particulière du signifiant et, de façon plus large, de ce qu'on identifie souvent comme les caractères de la modernité. On saura gré à l'auteure de nous avoir permis cette redécouverte des textes d'Anne Hébert.

1. Ottawa, Éditions de l'Université d'Ottawa, 1985, 192 p. 